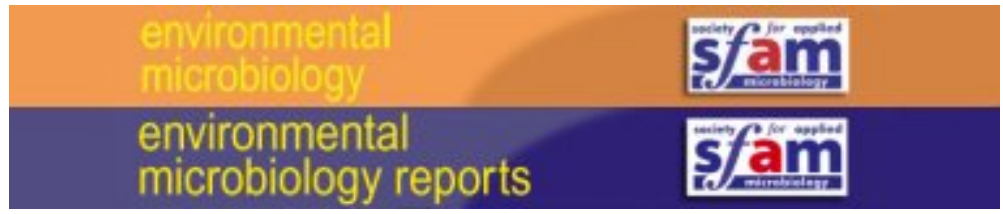

\title{
Evaluation of a new primer combination to minimize plastid contamination in 16S rDNA metabarcoding analyses of alga- associated bacterial communities
}

\begin{tabular}{|r|l|}
\hline Journal: & Environmental Microbiology and Environmental Microbiology Reports \\
\hline Manuscript ID & EMIR-2019-1373.R1 \\
\hline Journal: & Environmental Microbiology Reports \\
\hline Danuscript Type: & EMIR - Brief report \\
\hline Author: & n/a \\
\hline & $\begin{array}{l}\text { Thomas, François; Station Biologique de Roscoff, UMR8227 (CNRS- } \\
\text { Sorbonne Université) } \\
\text { Dittami, Simon; Station Biologique de Roscoff, UMR8227 (CNRS- } \\
\text { Sorbonne Université) } \\
\text { Brunet, Maéva; Station Biologique de Roscoff, UMR8227 (CNRS- } \\
\text { Sorbonne Université) } \\
\text { Le Duff, Nolwen; Station Biologique de Roscoff, UMR8227 (CNRS- } \\
\text { Sorbonne Université) } \\
\text { Tanguy, Gwenn; Station Biologique de Roscoff, FR2424, Genomer } \\
\text { Sequencing platform } \\
\text { Leblanc, Catherine; Station Biologique de Roscoff, LBI2M, UMR8227, } \\
\text { CNRS-UPMC } \\
\text { Gobet, Angélique ; MARBEC }\end{array}$ \\
\hline Keywords: & $\begin{array}{l}\text { chloroplast, plastid, 16S rDNA, alga-associated bacteria, metabarcoding, } \\
\text { V34 primer, algal holobiont }\end{array}$ \\
\hline
\end{tabular}

\section{SCHOLARONE \\ Manuscripts}


1 Evaluation of a new primer combination to minimize plastid contamination

2 in $16 \mathrm{~S}$ rDNA metabarcoding analyses of alga-associated bacterial

\section{3 communities}

4 François Thomas ${ }^{1 *}$, Simon M. Dittami ${ }^{1}$, Maéva Brunet ${ }^{1}$, Nolwen Le Duff ${ }^{1}$, Gwenn Tanguy²,

5 Catherine Leblanc ${ }^{1}$ and Angélique Gobet ${ }^{1,3^{*}}$

$6{ }^{1}$ Sorbonne Université, CNRS, Integrative Biology of Marine Models (LBI2M),

7 Station Biologique de Roscoff (SBR), 29680 Roscoff, France

$8 \quad{ }^{2}$ CNRS, Sorbonne Université, FR2424, Genomer, Station Biologique de Roscoff, 29680

9 Roscoff, France

$10{ }^{3}$ MARBEC, Ifremer, IRD, Université de Montpellier, CNRS, 34203 Sète, France

* Corresponding authors:

13 François Thomas

14 Marine Glycobiology group, UMR8227

15 Station Biologique de Roscoff

16 Place George Teissier

1729680 Roscoff, France

18 email: fthomas@,sb-roscoff.fr

19 Ph: +33256452148

20 Fax: +33298292324

Angélique Gobet IFREMER - UMR MARBEC

Avenue Jean Monnet CS 30171

34203 Sète, France

email: angelique.gobet@ifremer.fr

$\mathrm{Ph}:+33499573250$

Fax: +33499573294

Chloroplast, plastid, 16S rDNA, alga-associated bacteria, metabarcoding, V34 primer, algal holobiont 
37 Running title: Avoiding plastids in metabarcoding of algal microbiota

38 Originality-Significance statement

- Our aim was to minimize the amplification of plastidial 16S rDNA when analyzing alga-associated bacterial communities using metabarcoding.

- We compared the predicted coverage of five primer combinations in silico, including a universal primer set and four new sets designed to avoid amplification from plastids.

- Sequencing of a synthetic community showed similar performances for the universal primer set and one new combination.

- On natural alga-associated microbiota, the relative abundance of plastid sequences was reduced by $99-100 \%$ with the new primer set while the recovered bacterial richness was higher than with the universal primers given identical sequencing efforts and the community structure was maintained.

\section{Summary}

Plant- and alga-associated bacterial communities are generally described via $16 \mathrm{~S}$ rDNA metabarcoding using universal primers. As plastid genomes encode $16 \mathrm{~S}$ rDNA related to cyanobacteria, these datasets frequently contain $>90 \%$ plastidial sequences, and the bacterial diversity may be under-sampled. To overcome this limitation we evaluated in silico the taxonomic coverage for four primer combinations targeting the 16S rDNA V3-V4 region. They included a forward primer universal to Bacteria (S-D-Bact-0341-b-S-17) and four reverse primers designed to avoid plastid DNA amplification. The best primer combination 
60 (NOCHL) was compared to the universal primer set in the wet lab using a synthetic

61 community and samples from 3 macroalgal species. The proportion of plastid sequences was reduced by $99-100 \%$ with the NOCHL primers compared to the universal primers, irrespective of algal hosts, sample collection and extraction protocols. Additionally, the NOCHL primers yielded a higher richness while maintaining the community structure. As Planctomycetes, Verrucomicrobia, and Cyanobacteria were underrepresented (70-90\%) compared to universal primers, combining the NOCHL set with taxon-specific primers may be useful for a complete description of the alga-associated bacterial diversity. The NOCHL primers represent an innovation to study algal holobionts without amplifying host plastid sequences and may further be applied on other photosynthetic hosts.

\section{Introduction}

Numerous studies on algae-associated bacteria have highlighted their importance for health and physiology of the algal holobiont as well as for biomass degradation (Barott et al., 2011; Egan et al., 2013, 2014; Singh and Reddy, 2016). Bacteria can be abundant on macroalgal surfaces, ranging from $10^{6}$ to $10^{7}$ cells per $\mathrm{cm}^{2}$, and encompass a large phylogenetic diversity (van der Loos et al., 2019). These communities are distinct from bacterial assemblages found in the surrounding seawater or on inert surfaces (Burke et al., 2011; Stratil et al., 2014; Lemay et al., 2018). The composition of algae-associated bacterial communities has been shown to be host-specific and to vary depending on algal tissue, seasons, sampling sites and physiological status of the algae (Staufenberger et al., 2008; Lachnit et al., 2011; Miranda et al., 2013; Stratil et al., 2013; Zozaya-Valdes et al., 2015; Aires et al., 2016; Paix et al., 2019). A common approach to characterize these algae-associated communities is metabarcoding, whereby a selected variable region of the $16 \mathrm{~S}$ rDNA is sequenced using a primer set universal to Bacteria (e.g., forward: S-D-Bact-0341-b-S-17 / reverse: S-D-Bact-0785-a-A-21 
85

(Klindworth et al, 2013)) Due to the classical sampling strategies for macroalgae-associated microbiomes (e.g. algal surface scraping or tissue grinding (Bengtsson et al., 2012; Aires et al., 2016)), one major issue is that bacterial DNA samples are often contaminated with algal DNA. Primers with a large taxonomic coverage for Bacteria will tend to also amplify $16 \mathrm{~S}$ rDNA sequences from plastids. This is because plastid genomes, deriving from endosymbiosis events, still encode $16 \mathrm{~S}$ rDNA closely related to their cyanobacterial ancestors. Datasets obtained from brown algal field samples using common universal primers may contain over $90 \%$ of sequences affiliated to plastid DNA (Leblanc, personal data). This high proportion of plastidial sequences decreases the sequencing depth for bacterial epibionts and reduces the power of diversity analyses. One strategy to allow a large coverage of Bacteria while minimizing $16 \mathrm{~S}$ gene amplification from plastids is to design a primer in a region that differs between plastid sequences and those of Bacteria. Chelius and Triplett have identified such a region between the positions 783 and 799 of the 16S rDNA (following the $E$. coli numbering system) and they designed the 799F primer which includes four mismatches with chloroplasts to amplify Bacteria while avoiding chloroplast amplification from maize roots (Chelius and Triplett, 2001). The resulting primer was then used in combination with primer 1193R (amplification product $394 \mathrm{bp}$ ) in several studies on bacterial communities associated with plants (Sagaram et al., 2009; Bodenhausen et al., 2013) or macroalgae (Vieira et al., 2016; Aires et al., 2018; Serebryakova et al., 2018). However, this primer set still yielded up to $38 \%$ of sequences affiliated to chloroplasts (Sagaram et al., 2009). Several sets of primers including the $799 \mathrm{~F}$ primer with or without modifications were subsequently designed and tested to minimize plastid contamination on samples from plants and algae (Hanshew et al., 2013; Miranda et al., 2013; Aires et al., 2016). These primer sets target the V5-V8 or V5-V9 regions, yielding products of ca. 590 bp or $750 \mathrm{bp}$, respectively. Although suitable for the 454 pyrosequencing technology used in the latter studies, this fragment length 
110 is not compatible with the current Illumina v3 sequencing chemistry, which produces paired-

111 end reads of $2 \times 300 \mathrm{bp}$. Allowing for a recommended overlap of at least $50 \mathrm{bp}$, the total 112 product length for this technology should not exceed $500 \mathrm{bp}$. Our objective was to evaluate 113 primer combinations matching these criteria while minimizing plastid contamination in $16 \mathrm{~S}$ 114 rDNA metabarcoding analyses by: (i) comparing the efficiency of a primer set universal to 115 Bacteria and that of four primer combinations avoiding plastid amplification in silico, (ii) 116 comparing the efficiency of the best primer combination from the in silico results with that of 117 the universal primer set in the wet lab.

\section{Results and Discussion} \\ In silico evaluation of primer combinations to minimize the amplification of plastid}

\section{1 sequences}

122 We evaluated several sets of primers to amplify a $450 \mathrm{bp}$ fragment spanning the V3 and V4 123 regions of the $16 \mathrm{~S}$ rDNA while minimizing amplification of plastid sequences (Table S1).

124 These primer sets were designed by combining the universal forward primer S-D-Bact-0341125 b-S-17 (Klindworth et al., 2013) with reverse primers corresponding to the reverse 126 complement of four V5-V8 forward primers previously used in 454 pyrosequencing to 127 minimize chloroplast contamination (Hanshew et al., 2013). The four new primer 128 combinations (E. coli position 341 to 785 ) covers most of the region amplified by the original 129 V34 set (E. coli position 341 to 799), ensuring the comparability of data. The performance of 130 these different primer combinations was tested by running an in silico PCR on the SILVA 131 database ssu-132 with the RefNR sequence collection, using the online tool SILVA TestPrime 1321.0 (Klindworth et al., 2013). The universal V34 combination with the original reverse primer 133 S-D-Bact-0785-a-A-21 had an in silico predicted coverage for plastids (Table 1) ranging from $13457.3 \%$ to $88.3 \%$ with zero or 2 allowed mismatches, respectively. By contrast, three of the 
135 four new combinations (NOCHL, NOCHL3, and NOCHL6 but not NOCHL7, Table S1)

136 showed consistently low predicted coverage for plastids, even with 2 allowed mismatches

$137(2.7 \%, 1.1 \%$ and $1.1 \%$, respectively). The overall coverage of these three primer 138 combinations for all bacterial sequences remained high (79\% with no mismatch, $85-90 \%$ with 139 two mismatches). However, the NOCHL3 and NOCHL6 primer sets were predicted to 140 perform poorly on a number of phyla, including Planctomycetes (12\% coverage with two 141 mismatches) and Verrucomicrobia (17\% coverage), which are known to be part of alga142 associated microbial communities (Bengtsson and Øvreås, 2010; Lage and Bondoso, 2014; 143 Vollmers et al., 2017). For all taxa, the NOCHL combination had equal or better coverage 144 than the NOCHL3 and NOCHL6 combinations, notably for Planctomycetes (82.3\%) and 145 Verrucomicrobia (48.7\%). The NOCHL combination was therefore considered the most 146 promising candidate to minimize plastid contamination while maintaining the overall 147 bacterial diversity, and chosen to prepare Illumina-sequencing libraries.

\section{Comparison of primer performances in the wet lab}

150 Performances of the NOCHL and V34 primer combinations were compared in vitro using 151 different types of samples. As a positive control for the metabarcoding experiment, we 152 constructed a mock community based on the genomic DNA of pure bacterial isolates. 153 Genomic DNA was extracted from a total of 32 sequenced bacterial strains (Table S2) and 154 mixed in known proportions to assemble the mock community. Furthermore, the microbiota 155 associated with three brown algal species was sampled according to different approaches. The 156 filamentous brown alga Ectocarpus subulatus samples were collected $5 \mathrm{~km}$ North of the 157 Hopkins River Falls (Australia) and whole tissues were ground before DNA extraction. Blade 158 samples of Laminaria hyperborea sporophytes were collected at the coast off Roscoff 159 (Brittany, France) and soaked in a lysis buffer for DNA extraction. The blades of L. digitata 
160 sporophytes from Roscoff were swabbed for DNA extraction. All samplings were performed 161 in triplicate. Details on sampling and DNA extraction protocols are available in 162 Supplementary Information. Amplicon and library preparation were carried out following the 163 standard Illumina protocol (Illumina web page, 2018), including several negative controls, as 164 detailed in Supplementary Information. Both the NOCHL and V34 primer pairs successfully 165 amplified a ca. 450-bp fragment covering a similar region (V3-V4) of the bacterial 16S 166 rDNA. Sequencing libraries were prepared in parallel for each sample (nine algal samples, 167 one mock community, and six negative controls) using each primer pair. Sequencing was 168 carried out using one run of an Illumina Miseq yielding a total of 5,549,008 read pairs. 169 Sequences were deposited at the ENA under project accession number PRJEB33453. After 170 quality checks and removal of exogenous contaminations, low-quality sequences, and blanks 171 (see Supplementary Information), 2,784,253 assembled reads remained for data analysis. 172 Sequences were clustered, assigned to operational taxonomic units (OTUs) at 97\% identity, 173 taxonomically classified with RDP classifier (Wang et al., 2007) on the Silva SSU database 174 release 132 (Quast et al., 2013), and filtered to remove rare OTUs (see details in 175 Supplementary Information). The final dataset comprised 3,009 OTUs.

176 First, we compared metabarcoding results from the primer combinations V34 and NOCHL on 177 the mock community. The sequencing error rate calculated as in Kozich et al. (2013) from the 178 mock community datasets was $0.54 \%$ for both the V34 and NOCHL libraries. A total of 65 179 OTUs were detected in the rarefied mock community datasets. The expected relative 180 proportion $p_{i}$ of $16 \mathrm{~S}$ rDNAs from strain $i$ was calculated as follows: 


$$
p_{i}=\frac{\frac{q_{i}}{M_{i}} \times c_{i}}{\sum_{i=1}^{32} \frac{q_{i}}{M_{i}} \times c_{i}}
$$

where $q_{i}$ is the mass of DNA from strain $i$ added in the MOCK community, $M_{i}$ is the molecular weight of the complete genome for strain $i$ and $c_{i}$ is the number of $16 \mathrm{~S}$ rDNA gene copies in the genome of strain $i$. At the genus level, poor correlations (Pearson coefficient $<0.4)$ were obtained between the expected 16S rDNA proportions in the mock community and the relative abundance of taxa found in V34 or NOCHL libraries (Table 2). From the 26 genera represented in the mock community, 18 and 20 were detected in the V34 and NOCHL datasets, respectively. Both primer combinations did not detect the 6 genera Nonlabens, Agrococcus, Arthrobacter, Dokdonia, Roseovarius, and Imperialibacter. Sequences from these genera might either not be amplified by the primer sets, or more likely be merged to closely related sequences during OTU clustering. In addition, Roseobacter and Hoeflea were missed by the V34 primer combination, whereas their relative abundance was close to the expected value in the NOCHL dataset. With both primer pairs, ca. $30 \%$ of sequences could not be classified down to the genus level, leading to discrepancies between expected and measured proportions. A striking example is Vibrio: its expected abundance of $24.63 \%$ was largely underestimated at ca. $8 \%$ with both primer pairs, whereas $11 \%$ of sequences were assigned to unclassified Vibrionaceae. On the contrary, both primer combinations largely overestimated the proportion of Pseudoalteromonas, possibly due to the proximity with sequences from Alteromonas (Gauthier et al., 1995). Although the NOCHL combination had a suboptimal predicted coverage for Planctomycetes (Table 1), it still detected the planctomycetal genus Rhodopirellula in the mock community, underestimating it by $56 \%$. On the other hand, the V34 combination overestimated Rhodopirellula by 47\%. As expected, correlations between theoretical and measured proportions were much better (Pearson coefficient $>0.9$ ) at the family level for both primer combinations (Table S3). All 13 families 
represented in the mock community were detected using the NOCHL combination, whereas

209 the V34 combination missed the Phyllobacteriaceae family. Altogether, the performance of

210 the NOCHL combination was found to be comparable to the V34 set on the mock community

211 in terms of sensitivity and accuracy.

213 We further compared the efficiency of the primer combinations V34 and NOCHL in field 214 samples, characterizing the bacterial community associated with Ectocarpus subulatus 215 (ECTO), Laminaria digitata (LDIG) and Laminaria hyperborea (LHYP). The relative 216 abundance of sequences affiliated to plastids ranged from $0.6 \%$ to $66 \%$ in the V34 dataset 217 (Figure 1). The highest relative abundances of plastid sequences were found when algal 218 tissues were ground (ECTO) or soaked in lysis buffer (LHYP) before DNA extraction, 219 compared to the swab-based technique (LDIG). It was drastically reduced by $99-100 \%$ with 220 the NOCHL combination in all three types of alga-associated samples analyzed (Figure 1), 221 with relative abundance of plastid $16 \mathrm{~S}$ rDNA sequences ranging from 0 to $0.3 \%$. This 222 confirms that the new primer combination succeeds at minimizing plastid contamination, 223 irrespective of the algal host, sample collection and DNA extraction protocol. The NOCHL 224 combination was more efficient at reducing plastid contamination compared to a previous 225 Illumina metabarcoding analysis of Ectocarpus-associated bacterial communities. In that 226 former study, a modified set of $341 \mathrm{~F}$ and $806 \mathrm{R}$ primers still yielded $32 \%$ of plastid sequences 227 despite a central mismatch to avoid plastid DNA amplification (Dittami et al., 2016).

228 Alpha-diversity analyses tended to show higher values of OTU richness (observed OTUs) and 229 diversity (Shannon and Simpson indices) with the NOCHL combination compared to V34 230 before removal of plastid sequences (Figure 2A). This overall effect was found significant for 231 Shannon and Simpson diversity indices and was more pronounced for samples from $E$. 232 subulatus and L. hyperborea, After removal of plastid sequences (Figure 2B), the NOCHL 
233 primer combination yielded significantly higher richness than V34 (Student's t-test, $\mathrm{P}=0.05$ )

234 with an overall increase of $10 \%$. Again, this effect was more pronounced for ECTO and

235 LHYP samples. Indeed, ECTO and LHYP samples, where plastid contamination was the

236 highest (Figure 1), showed the strongest effect of plastidial sequence removal on sequencing 237 depth (Figure S1). This exemplifies the issue faced with universal primers, where a large 238 amount of plastid-affiliated sequences can decrease the sequencing depth for target bacterial 239 sequences and reduce the evenness. After removal of plastid sequences, lower Shannon and 240 Simpson diversity indices were detected with the NOCHL combination compared with V34 ($2413.2 \%$ and $-0.5 \%$ lower values for Shannon and Simpson, respectively). This was mostly due 242 to an increase in these indices for V34 datasets after removal of plastid sequences, while they 243 stayed stable for the NOCHL datasets. Although not the main focus of this work, we noticed 244 that bacterial communities retrieved from Ectocarpus were more diverse than those from the 245 two Laminaria species. This might be due to the nature of the samples as well as the 246 extraction protocol where entire algal specimens were ground for Ectocarpus (i.e. including 247 the endomicrobiota), while for Laminaria the extraction protocols only targeted surface248 attached bacteria. Joint hierarchical clustering of OTU-level datasets for both primer pairs 249 after removal of plastid sequences showed that samples grouped according to algal host rather 250 than primer pair (Figure 3A).

251 The relative abundance of phyla was generally similar between paired sets of samples (Figure 252 3B). Notable exceptions were the Planctomycetes, Verrucomicrobia, and Cyanobacteria that 253 had lower relative abundance in the NOCHL libraries compared to V34 (Table S4). We 254 further searched for differential OTUs between pairs of samples sequenced with the V34 or 255 NOCHL combination. This analysis was performed with the edgeR package (Robinson et al., 256 2009) on the non-transformed dataset after removal of plastid sequences, and accounted for 257 the paired design of the study, i.e. the same samples were sequenced with the two primer 
258 combinations. The significance threshold was set at $\alpha=5 \%$ after Benjamini-Hochberg 259 correction for multiple testing. Out of the 2,852 OTUs remaining after discarding plastids, the 260 edgeR analysis detected 39 differential OTUs (i.e. 1.4\%) between primer combinations (Table

261 S5). All differential OTUs were more abundant in the V34-amplified dataset compared to 262 NOCHL. The majority of them belonged to Verrucomicrobiae and Planctomycetacia (Figure 263 4), partly reflecting the lower predicted coverage of NOCHL for these taxa. The apparent 264 lower abundance of Plantomycetacia OTUs using the NOCHL primer set could also be in 265 part due to their over-estimation with the V34 combination, as shown in the mock community 266 analysis (Table 2). The highest fold-change was found for OTU00082 belonging to 267 Cyanobacteria. The lower abundance of Cyanobacteria was inevitable since plastids and 268 extant cyanobacteria share a common ancestor and therefore have homologous 16S rDNA 269 sequences (Giovannoni et al., 1988; Delwiche, 1999).

\section{Conclusion}

272 Considering that plastid sequences can represent more than $90 \%$ of all sequences in one 273 sample when using universal primer sets, the bacterial diversity in a sample may be 274 underrepresented. The new primer set NOCHL is efficient to avoid amplifying plastid 275 sequences from an algal host while identifying a significantly higher bacterial richness than 276 with the universal primers V34, given identical sequencing efforts. This validates that fewer 277 plastid sequences in the samples lead to a larger access to bacterial sequences thus recovering 278 more of the bacterial diversity. However, as some bacterial groups may be underrepresented, 279 this primer set may be combined with taxon-specific primers (e.g. for Planctomycetes) for a 280 more complete coverage of Bacteria. Still, the data obtained with the NOCHL and universal 281 V34 primer sets are comparable since the targeted regions are the same and patterns of the community structure are similar. Although validated here on macroalgal samples, this primer 
283 set may be useful to characterize bacterial communities from other environments where

284 plastid contamination can be an issue such as terrestrial plants, microalgae, or gut microbiota 285 of herbivores.

\section{Acknowledgments and funding}

288 The authors have no conflict of interest to declare. We warmly thank Gaëlle Correc and 289 Sylvie Rousvoal for help with sampling as well as Florian de Bettignies and Dominique 290 Davoult for providing samples. We thank Frédérique Le Roux and Christian Jeanthon for 291 providing bacterial strains. This work has benefited from the facilities of the Genomer 292 platform and from the computational resources of the ABiMS bioinformatics platform (FR 293 2424, CNRS-Sorbonne Université, Roscoff), which are part of the Biogenouest core facility 294 network.

295 This work has benefited from the support of the French Government via the National 296 Research Agency investment expenditure program IDEALG (ANR-10-BTBR-04). FT, SD, 297 and CL acknowledge support by the Centre National de la Recherche Scientifique (CNRS). 298 AG acknowledges support by the Institut Français de Recherche pour l'Exploitation de la Mer 299 (IFREMER).

\section{References}

302 Aires, T., Muyzer, G., Serrão, E.A., and Engelen, A.H. (2018) Unraveling seaweeds 303 bacteriomes. In, Charrier,B., Wichard,T., and Reddy,C.R.K. (eds), Protocols for $304 \quad$ Macroalgae Research. CRC Press, pp. 95-113.

305 Aires, T., Serrão, E.A., and Engelen, A.H. (2016) Host and environmental specificity in bacterial communities associated to two highly invasive marine species (genus Asparagopsis). Front Microbiol 7: 1-14. 
308 Barott, K.L., Rodriguez-Brito, B., Janouškovec, J., Marhaver, K.L., Smith, J.E., Keeling, P., and Rohwer, F.L. (2011) Microbial diversity associated with four functional groups of benthic reef algae and the reef-building coral Montastraea annularis. Environ Microbiol 13: 1192-1204.

Bengtsson, M.M. and Øvreås, L. (2010) Planctomycetes dominate biofilms on surfaces of the kelp Laminaria hyperborea. BMC Microbiol 10: 261.

314 Bengtsson, M.M., Sjøtun, K., Lanzén, A., and Øvreås, L. (2012) Bacterial diversity in relation to secondary production and succession on surfaces of the kelp Laminaria hyperborea. ISME J 6: 2188-2198.

Bodenhausen, N., Horton, M.W., and Bergelson, J. (2013) Bacterial Communities Associated with the Leaves and the Roots of Arabidopsis thaliana. PLoS One 8:.

Burke, C., Thomas, T., Lewis, M., Steinberg, P., and Kjelleberg, S. (2011) Composition, uniqueness and variability of the epiphytic bacterial community of the green alga Ulva australis. ISME J 5: 590-600.

Chelius, M.K. and Triplett, E.W. (2001) The diversity of archaea and bacteria in association with the roots of Zea mays L. Microb Ecol 41: 252-263.

Delwiche (1999) Tracing the thread of plastid diversity through the tapestry of life. Am Nat 154: S164.

Dittami, S.M., Duboscq-Bidot, L., Perennou, M., Gobet, A., Corre, E., Boyen, C., and Tonon, T. (2016) Host-microbe interactions as a driver of acclimation to salinity gradients in brown algal cultures. ISME J 10: 51-63.

Egan, S., Fernandes, N.D., Kumar, V., Gardiner, M., and Thomas, T. (2014) Bacterial pathogens, virulence mechanism and host defence in marine macroalgae. Environ Microbiol 16: 925-938.

332 Egan, S., Harder, T., Burke, C., Steinberg, P., Kjelleberg, S., and Thomas, T. (2013) The 

seaweed holobiont: Understanding seaweed-bacteria interactions. FEMS Microbiol Rev 37: 462-476.

Gauthier, G., Gauthier, M., and Christen, R. (1995) Phylogenetic analysis of the genera Alteromonas, Shewanella, and Moritella using genes coding for small-subunit rRNA sequences and division of the genus Alteromonas into two genera, Alteromonas (emended) and Pseudoalteromonas gen. nov., and proposal of twelve new species combinations. Int J Syst Bacteriol 45: 755-761.

Giovannoni, S.J., Turner, S., Olsen, G.J., Barns, S., Lane, D.J., and Pace, N.R. (1988) Evolutionary relationships among cyanobacteria and green chloroplasts. J Bacteriol 170: $3584-3592$.

Hanshew, A.S., Mason, C.J., Raffa, K.F., and Currie, C.R. (2013) Minimization of chloroplast contamination in 16S rRNA gene pyrosequencing of insect herbivore bacterial communities. J Microbiol Methods 95: 149-155.

Illumina web page (2018) 16S Metagenomic Sequencing Library Preparation.

Klindworth, A., Pruesse, E., Schweer, T., Peplies, J., Quast, C., Horn, M., and Glöckner, F.O. (2013) Evaluation of general 16S ribosomal RNA gene PCR primers for classical and next-generation sequencing-based diversity studies. Nucleic Acids Res 41: 1-11.

Kozich, J.J., Westcott, S.L., Baxter, N.T., Highlander, S.K., and Schloss, P.D. (2013) Development of a dual-index sequencing strategy and curation pipeline for analyzing amplicon sequence data on the MiSeq Illumina sequencing platform. Appl Environ Microbiol 79: 5112-20.

Lachnit, T., Meske, D., Wahl, M., Harder, T., and Schmitz, R. (2011) Epibacterial community patterns on marine macroalgae are host-specific but temporally variable. Environ Microbiol 13: 655-665.

Lage, O.M. and Bondoso, J. (2014) Planctomycetes and macroalgae, a striking association. 
359 Lemay, M., Martone, P., Keeling, P., Burt, J., Krumhansl, K., Sanders, R., and Parfrey, L. (2018) Sympatric kelp species share a large portion of their surface bacterial communities. Environ Microbiol 20: 658-670.

van der Loos, L.M., Eriksson, B.K., and Falcão Salles, J. (2019) The macroalgal holobiont in a changing sea. Trends Microbiol 27: 635-650.

364 Miranda, L.N., Hutchison, K., Grossman, A.R., and Brawley, S.H. (2013) Diversity and abundance of the bacterial community of the red macroalga Porphyra umbilicalis: Did bacterial farmers produce macroalgae? PLoS One 8: e58269.

Paix, B., Othmani, A., Debroas, D., Culioli, G., and Briand, J.F. (2019) Temporal covariation of epibacterial community and surface metabolome in the Mediterranean seaweed holobiont Taonia atomaria. Environ Microbiol. doi: 10.1111/1462-2920.14617

Quast, C., Pruesse, E., Yilmaz, P., Gerken, J., Schweer, T., Yarza, P., et al. (2013) The SILVA ribosomal RNA gene database project: improved data processing and web-based tools. Nucleic Acids Res 41: D590-6.

Robinson, M.D., McCarthy, D.J., and Smyth, G.K. (2009) edgeR: A Bioconductor package for differential expression analysis of digital gene expression data. Bioinformatics 26: 139-140.

Sagaram, U.S., Deangelis, K.M., Trivedi, P., Andersen, G.L., Lu, S.E., and Wang, N. (2009) Bacterial diversity analysis of huanglongbing pathogen-infected citrus, using phyloChip arrays and 16S rRNA gene clone library sequencing. Appl Environ Microbiol 75: 15661574.

Serebryakova, A., Aires, T., Viard, F., Serrão, E.A., and Engelen, A.H. (2018) Summer shifts of bacterial communities associated with the invasive brown seaweed Sargassum muticum are location and tissue dependent. PLoS One 13: 1-18. 
383 Singh, R.P. and Reddy, C.R.K. (2016) Unraveling the functions of the macroalgal microbiome. Front Microbiol 6: 1-8.

385

Staufenberger, T., Thiel, V., Wiese, J., and Imhoff, J.F. (2008) Phylogenetic analysis of bacteria associated with Laminaria saccharina. FEMS Microbiol Ecol 64: 65-77.

Stratil, S.B., Neulinger, S.C., Knecht, H., Friedrichs, A.K., and Wahl, M. (2014) Salinity affects compositional traits of epibacterial communities on the brown macroalga Fucus vesiculosus. FEMS Microbiol Ecol 88: 272-279.

Stratil, S.B., Neulinger, S.C., Knecht, H., Friedrichs, A.K., and Wahl, M. (2013) Temperature-driven shifts in the epibiotic bacterial community composition of the brown macroalga Fucus vesiculosus. Microbiologyopen 2: 338-349.

Vieira, C., Engelen, A.H., Guentas, L., Aires, T., Houlbreque, F., Gaubert, J., et al. (2016) Species specificity of bacteria associated to the brown seaweeds Lobophora (Dictyotales, Phaeophyceae) and their potential for induction of rapid coral bleaching in Acropora muricata. Front Microbiol 7: 1-13.

Vollmers, J., Frentrup, M., Rast, P., Jogler, C., and Kaster, A.K. (2017) Untangling genomes of novel Planctomycetal and Verrucomicrobial species from Monterey bay kelp forest metagenomes by refined binning. Front Microbiol $\mathbf{8}$ :.

Wang, Q., Garrity, G.M., Tiedje, J.M., and Cole, J.R. (2007) Naive Bayesian classifier for rapid assignment of rRNA sequences into the new bacterial taxonomy. Appl Environ Microbiol 73: 5261-7.

Zozaya-Valdes, E., Egan, S., and Thomas, T. (2015) A comprehensive analysis of the microbial communities of healthy and diseased marine macroalgae and the detection of known and potential bacterial pathogens. Front Microbiol 6: 1-9. 


\section{$\underline{\text { Table and Figure legends }}$}

411 Figure 1: Relative abundance of sequences affiliated to plastid OTUs using the V34 or

412 NOCHL primer sets. Bacterial communities were analyzed in triplicate samples from 413 different algal hosts: ECTO, Ectocarpus subulatus; LHYP, Laminaria hyperborea; LDIG, 414 Laminaria digitata.

Figure 2: Richness and diversity estimates before (A) and after (B) removal of OTUs affiliated to plastids. Data were subsampled at 8,358 sequences per sample prior to analysis.

418 Observed, observed OTU richness; Shannon, Shannon index; Simpson, Simpson index;

419 ECTO, Ectocarpus subulatus; LDIG, Laminaria digitata; LHYP, Laminaria hyperborea.

420 Results of paired Student's t-test testing the effect of the set of primers on each measured

421 estimate are reported below each panel $(\mathrm{df}=8)$.

Figure 3: Effect of the primer pair on the detected bacterial community composition, after removal of OTUs affiliated to plastids.

(A) Hierarchical clustering calculated on Hellinger-transformed data based on Morisita-Horn distance using the complete linkage algorithm

(B) Taxonomic composition based on the phylum level

Figure 4: Taxonomic affiliation of the 39 differential OTUs detected with edgeR, depending on the primer pair. All of these OTUs received higher coverage with the V34 primers.

431 Supplementary Figure 1: Rarefaction analysis of datasets obtained with V34 and NOCHL primer sets before (A) and after (B) removal of plastid-related sequences. 
434 Table 1: In silico predicted coverage of selected bacterial taxa, relative to the sequences

435 available in the Silva SSU r132 database for different primer combinations using TestPrime

436 1.0. A length of 3 bases of 0 -mismatch zone at 3' end of primers was chosen for tests with two

437 allowed mismatches in the sequence.

438

439 Table 2: Genus-level comparison of theoretical relative abundance in the mock community

440 with the measured abundance in libraries sequenced with V34 or NOCHL primers. unclass.,

441 unclassified.

442 
1 Evaluation of a new primer combination to minimize plastid contamination

2 in $16 \mathrm{~S}$ rDNA metabarcoding analyses of alga-associated bacterial

\section{3 communities}

4 François Thomas ${ }^{1 *}$, Simon M. Dittami ${ }^{1}$, Maéva Brunet ${ }^{1}$, Nolwen Le Duff ${ }^{1}$, Gwenn Tanguy²,

5 Catherine Leblanc ${ }^{1}$ and Angélique Gobet ${ }^{1,3^{*}}$

$6{ }^{1}$ Sorbonne Université, CNRS, Integrative Biology of Marine Models (LBI2M),

7 Station Biologique de Roscoff (SBR), 29680 Roscoff, France

$8 \quad{ }^{2}$ CNRS, Sorbonne Université, FR2424, Genomer, Station Biologique de Roscoff, 29680

9 Roscoff, France

$10{ }^{3}$ MARBEC, Ifremer, IRD, Université de Montpellier, CNRS, 34203 Sète, France

11

$12 *$ Corresponding authors:

13 François Thomas

14 Marine Glycobiology group, UMR8227

15 Station Biologique de Roscoff

16 Place George Teissier

1729680 Roscoff, France

18 email: fthomas@,sb-roscoff.fr

19 Ph: +33256452148

20 Fax: +33298292324

Angélique Gobet IFREMER - UMR MARBEC

Avenue Jean Monnet CS 30171

34203 Sète, France

email: angelique.gobet $@$,ifremer.fr

Ph: +33499573250

Fax: +33499573294

Chloroplast, plastid, 16S rDNA, alga-associated bacteria, metabarcoding, V34 primer, algal holobiont 
37 Running title: Avoiding plastids in metabarcoding of algal microbiota

38 Originality-Significance statement

- Our aim was to minimize the amplification of plastidial 16S rDNA when analyzing alga-associated bacterial communities using metabarcoding.

- We compared the predicted coverage of five primer combinations in silico, including a universal primer set and four new sets designed to avoid amplification from plastids.

- Sequencing of a synthetic community showed similar performances for the universal primer set and one new combination.

- On natural alga-associated microbiota, the relative abundance of plastid sequences was reduced by $99-100 \%$ with the new primer set while the recovered bacterial richness was higher than with the universal primers given identical sequencing efforts and the community structure was maintained.

\section{Summary}

Plant- and alga-associated bacterial communities are generally described via $16 \mathrm{~S}$ rDNA metabarcoding using universal primers. As plastid genomes encode $16 \mathrm{~S}$ rDNA related to cyanobacteria, these datasets frequently contain $>90 \%$ plastidial sequences, and the bacterial diversity may be under-sampled. To overcome this limitation we evaluated in silico the taxonomic coverage for four primer combinations targeting the 16S rDNA V3-V4 region. They included a forward primer universal to Bacteria (S-D-Bact-0341-b-S-17) and four reverse primers designed to avoid plastid DNA amplification. The best primer combination 
60 (NOCHL) was compared to the universal primer set in the wet lab using a synthetic community and samples from 3 macroalgal species. The proportion of plastid sequences was reduced by $99-100 \%$ with the NOCHL primers compared to the universal primers, irrespective of algal hosts, sample collection and extraction protocols. Additionally, the NOCHL primers yielded a higher richness while maintaining the community structure. As Planctomycetes, Verrucomicrobia, and Cyanobacteria were underrepresented (70-90\%) compared to universal primers, combining the NOCHL set with taxon-specific primers may be useful for a complete description of the alga-associated bacterial diversity. The NOCHL primers represent an innovation to study algal holobionts without amplifying host plastid sequences and may further be applied on other photosynthetic hosts.

\section{Introduction}

Numerous studies on algae-associated bacteria have highlighted their importance for health and physiology of the algal holobiont as well as for biomass degradation (Barott et al., 2011; Egan et al., 2013, 2014; Singh and Reddy, 2016). Bacteria can be abundant on macroalgal surfaces, ranging from $10^{6}$ to $10^{7}$ cells per $\mathrm{cm}^{2}$, and encompass a large phylogenetic diversity (van der Loos et al., 2019). These communities are distinct from bacterial assemblages found in the surrounding seawater or on inert surfaces (Burke et al., 2011; Stratil et al., 2014; Lemay et al., 2018). The composition of algae-associated bacterial communities has been shown to be host-specific and to vary depending on algal tissue, seasons, sampling sites and physiological status of the algae (Staufenberger et al., 2008; Lachnit et al., 2011; Miranda et al., 2013; Stratil et al., 2013; Zozaya-Valdes et al., 2015; Aires et al., 2016; Paix et al., 2019). A common approach to characterize these algae-associated communities is metabarcoding, whereby a selected variable region of the $16 \mathrm{~S}$ rDNA is sequenced using a primer set universal 
85

(Klindworth et al, 2013)) Due to the classical sampling strategies for macroalgae-associated microbiomes (e.g. algal surface scraping or tissue grinding (Bengtsson et al., 2012; Aires et al., 2016)), one major issue is that bacterial DNA samples are often contaminated with algal DNA. Primers with a large taxonomic coverage for Bacteria will tend to also amplify $16 \mathrm{~S}$ rDNA sequences from plastids. This is because plastid genomes, deriving from endosymbiosis events, still encode 16S rDNA closely related to their cyanobacterial ancestors. Datasets obtained from brown algal field samples using common universal primers may contain over $90 \%$ of sequences affiliated to plastid DNA (Leblanc, personal data). This high proportion of plastidial sequences decreases the sequencing depth for bacterial epibionts and reduces the power of diversity analyses. One strategy to allow a large coverage of Bacteria while minimizing $16 \mathrm{~S}$ gene amplification from plastids is to design a primer in a region that differs between plastid sequences and those of Bacteria. Chelius and Triplett have identified such a region between the positions 783 and 799 of the 16S rDNA (following the $E$. coli numbering system) and they designed the $799 \mathrm{~F}$ primer which includes four mismatches with chloroplasts to amplify Bacteria while avoiding chloroplast amplification from maize roots (Chelius and Triplett, 2001). The resulting primer was then used in combination with primer 1193R (amplification product $394 \mathrm{bp}$ ) in several studies on bacterial communities associated with plants (Sagaram et al., 2009; Bodenhausen et al., 2013) or macroalgae (Vieira et al., 2016; Aires et al., 2018; Serebryakova et al., 2018). However, this primer set still yielded up to $38 \%$ of sequences affiliated to chloroplasts (Sagaram et al., 2009). Several sets of primers including the $799 \mathrm{~F}$ primer with or without modifications were subsequently designed and tested to minimize plastid contamination on samples from plants and algae (Hanshew et al., 2013; Miranda et al., 2013; Aires et al., 2016). These primer sets target the V5-V8 or V5-V9 regions, yielding products of ca. 590 bp or $750 \mathrm{bp}$, respectively. Although suitable for the 454 pyrosequencing technology used in the latter studies, this fragment length 
110 is not compatible with the current Illumina v3 sequencing chemistry, which produces paired-

111 end reads of $2 \times 300 \mathrm{bp}$. Allowing for a recommended overlap of at least $50 \mathrm{bp}$, the total 112 product length for this technology should not exceed $500 \mathrm{bp}$. Our objective was to evaluate 113 primer combinations matching these criteria while minimizing plastid contamination in $16 \mathrm{~S}$ 114 rDNA metabarcoding analyses by: (i) comparing the efficiency of a primer set universal to 115 Bacteria and that of four primer combinations avoiding plastid amplification in silico, (ii) 116 comparing the efficiency of the best primer combination from the in silico results with that of 117 the universal primer set in the wet lab.

\section{Results and Discussion}

\section{In silico evaluation of primer combinations to minimize the amplification of plastid}

\section{1 sequences}

122 We evaluated several sets of primers to amplify a 450 bp fragment spanning the V3 and V4 123 regions of the $16 \mathrm{~S}$ rDNA while minimizing amplification of plastid sequences (Table S1).

124 These primer sets were designed by combining the universal forward primer S-D-Bact-0341125 b-S-17 (Klindworth et al., 2013) with reverse primers corresponding to the reverse 126 complement of four V5-V8 forward primers previously used in 454 pyrosequencing to 127 minimize chloroplast contamination (Hanshew et al., 2013). The four new primer 128 combinations (E. coli position 341 to 785 ) covers most of the region amplified by the original 129 V34 set (E. coli position 341 to 799 ), ensuring the comparability of data. The performance of 130 these different primer combinations was tested by running an in silico PCR on the SILVA 131 database ssu-132 with the RefNR sequence collection, using the online tool SILVA TestPrime 1321.0 (Klindworth et al., 2013). The universal V34 combination with the original reverse primer 133 S-D-Bact-0785-a-A-21 had an in silico predicted coverage for plastids (Table 1) ranging from $13457.3 \%$ to $88.3 \%$ with zero or 2 allowed mismatches, respectively. By contrast, three of the 
135 four new combinations (NOCHL, NOCHL3, and NOCHL6 but not NOCHL7, Table S1)

136 showed consistently low predicted coverage for plastids, even with 2 allowed mismatches

$137(2.7 \%, 1.1 \%$ and $1.1 \%$, respectively). The overall coverage of these three primer 138 combinations for all bacterial sequences remained high (79\% with no mismatch, $85-90 \%$ with 139 two mismatches). However, the NOCHL3 and NOCHL6 primer sets were predicted to 140 perform poorly on a number of phyla, including Planctomycetes (12\% coverage with two 141 mismatches) and Verrucomicrobia (17\% coverage), which are known to be part of alga142 associated microbial communities (Bengtsson and Øvreås, 2010; Lage and Bondoso, 2014; 143 Vollmers et al., 2017). For all taxa, the NOCHL combination had equal or better coverage 144 than the NOCHL3 and NOCHL6 combinations, notably for Planctomycetes (82.3\%) and 145 Verrucomicrobia (48.7\%). The NOCHL combination was therefore considered the most 146 promising candidate to minimize plastid contamination while maintaining the overall 147 bacterial diversity, and chosen to prepare Illumina-sequencing libraries.

\section{Comparison of primer performances in the wet lab}

150 Performances of the NOCHL and V34 primer combinations were compared in vitro using 151 different types of samples. As a positive control for the metabarcoding experiment, we constructed a mock community based on the genomic DNA of pure bacterial isolates.

153 Genomic DNA was extracted from a total of 32 sequenced bacterial strains (Table S2) and 154 mixed in known proportions to assemble the mock community. Furthermore, the microbiota 155 associated with three brown algal species was sampled according to different approaches. The 156 filamentous brown alga Ectocarpus subulatus samples were collected $5 \mathrm{~km}$ North of the 157 Hopkins River Falls (Australia) and whole tissues were ground before DNA extraction. Blade samples of Laminaria hyperborea sporophytes were collected at the coast off Roscoff 159 (Brittany, France) and soaked in a lysis buffer for DNA extraction. The blades of L. digitata 
160 sporophytes from Roscoff were swabbed for DNA extraction. All samplings were performed 161 in triplicate. Details on sampling and DNA extraction protocols are available in 162 Supplementary Information. Amplicon and library preparation were carried out following the 163 standard Illumina protocol (Illumina web page, 2018), including several negative controls, as 164 detailed in Supplementary Information. Both the NOCHL and V34 primer pairs successfully 165 amplified a ca. 450-bp fragment covering a similar region (V3-V4) of the bacterial 16S 166 rDNA. Sequencing libraries were prepared in parallel for each sample (nine algal samples, 167 one mock community, and six negative controls) using each primer pair. Sequencing was 168 carried out using one run of an Illumina Miseq yielding a total of 5,549,008 read pairs. 169 Sequences were deposited at the ENA under project accession number PRJEB33453. After 170 quality checks and removal of exogenous contaminations, low-quality sequences, and blanks 171 (see Supplementary Information), 2,784,253 assembled reads remained for data analysis. 172 Sequences were clustered, assigned to operational taxonomic units (OTUs) at 97\% identity, 173 taxonomically classified with RDP classifier (Wang et al., 2007) on the Silva SSU database 174 release 132 (Quast et al., 2013), and filtered to remove rare OTUs (see details in 175 Supplementary Information). The final dataset comprised 3,009 OTUs.

176 First, we compared metabarcoding results from the primer combinations V34 and NOCHL on 177 the mock community. The sequencing error rate calculated as in Kozich et al. (2013) from the 178 mock community datasets was $0.54 \%$ for both the V34 and NOCHL libraries. A total of 65 179 OTUs were detected in the rarefied mock community datasets. The expected relative 180 proportion $p_{i}$ of $16 \mathrm{~S}$ rDNAs from strain $i$ was calculated as follows: 


$$
p_{i}=\frac{\frac{q_{i}}{M_{i}} \times c_{i}}{\sum_{i=1}^{32} \frac{q_{i}}{M_{i}} \times c_{i}}
$$

where $q_{i}$ is the mass of DNA from strain $i$ added in the MOCK community, $M_{i}$ is the molecular weight of the complete genome for strain $i$ and $c_{i}$ is the number of 16S rDNA gene copies in the genome of strain $i$. At the genus level, poor correlations (Pearson coefficient $<0.4)$ were obtained between the expected 16S rDNA proportions in the mock community and the relative abundance of taxa found in V34 or NOCHL libraries (Table 2). From the 26 genera represented in the mock community, 18 and 20 were detected in the V34 and NOCHL datasets, respectively. Both primer combinations did not detect the 6 genera Nonlabens, Agrococcus, Arthrobacter, Dokdonia, Roseovarius, and Imperialibacter. Sequences from these genera might either not be amplified by the primer sets, or more likely be merged to closely related sequences during OTU clustering. In addition, Roseobacter and Hoeflea were missed by the V34 primer combination, whereas their relative abundance was close to the expected value in the NOCHL dataset. With both primer pairs, ca. $30 \%$ of sequences could not be classified down to the genus level, leading to discrepancies between expected and measured proportions. A striking example is Vibrio: its expected abundance of $24.63 \%$ was largely underestimated at ca. $8 \%$ with both primer pairs, whereas $11 \%$ of sequences were assigned to unclassified Vibrionaceae. On the contrary, both primer combinations largely overestimated the proportion of Pseudoalteromonas, possibly due to the proximity with sequences from Alteromonas (Gauthier et al., 1995). Although the NOCHL combination had a suboptimal predicted coverage for Planctomycetes (Table 1), it still detected the planctomycetal genus Rhodopirellula in the mock community, underestimating it by $56 \%$. On the other hand, the V34 combination overestimated Rhodopirellula by 47\%. As expected, correlations between theoretical and measured proportions were much better (Pearson coefficient $>0.9$ ) at the family level for both primer combinations (Table S3). All 13 families 
208 represented in the mock community were detected using the NOCHL combination, whereas 209 the V34 combination missed the Phyllobacteriaceae family. Altogether, the performance of 210 the NOCHL combination was found to be comparable to the V34 set on the mock community 211 in terms of sensitivity and accuracy.

213 We further compared the efficiency of the primer combinations V34 and NOCHL in field 214 samples, characterizing the bacterial community associated with Ectocarpus subulatus 215 (ECTO), Laminaria digitata (LDIG) and Laminaria hyperborea (LHYP). The relative 216 abundance of sequences affiliated to plastids ranged from $0.6 \%$ to $66 \%$ in the V34 dataset 217 (Figure 1). The highest relative abundances of plastid sequences were found when algal 218 tissues were ground (ECTO) or soaked in lysis buffer (LHYP) before DNA extraction, 219 compared to the swab-based technique (LDIG). It was drastically reduced by $99-100 \%$ with 220 the NOCHL combination in all three types of alga-associated samples analyzed (Figure 1), 221 with relative abundance of plastid $16 \mathrm{~S}$ rDNA sequences ranging from 0 to $0.3 \%$. This 222 confirms that the new primer combination succeeds at minimizing plastid contamination, 223 irrespective of the algal host, sample collection and DNA extraction protocol. The NOCHL 224 combination was more efficient at reducing plastid contamination compared to a previous 225 Illumina metabarcoding analysis of Ectocarpus-associated bacterial communities. In that 226 former study, a modified set of $341 \mathrm{~F}$ and $806 \mathrm{R}$ primers still yielded $32 \%$ of plastid sequences 227 despite a central mismatch to avoid plastid DNA amplification (Dittami et al., 2016).

228 Alpha-diversity analyses tended to show higher values of OTU richness (observed OTUs-and 229 Chao1 richness estimator) and diversity (Shannon and Simpson indices) with the NOCHL 230 combination compared to V34 before removal of plastid sequences (Figure 2A). This overall

231 effect was found significant for Shannon and Simpson diversity indices and was more 232 pronounced for samples from E. subulatus and L. hyperborea, After removal of plastid 
233 sequences (Figure 2B), the NOCHL primer combination yielded significantly higher richness 234 estimates-than V34 (Student's t-test, $\mathrm{P}=0.05$ ) with an overall increase of $10 \%$. Again, this 235 effect was more pronounced for ECTO and LHYP samples. Indeed, ECTO and LHYP 236 samples, where plastid contamination was the highest (Figure 1), showed the strongest effect 237 of plastidial sequence removal on sequencing depth (Figure S1). This exemplifies the issue 238 faced with universal primers, where a large amount of plastid-affiliated sequences can 239 decrease the sequencing depth for target bacterial sequences and reduce the evenness. After 240 removal of plastid sequences, lower Shannon and Simpson diversity indices were detected 241 with the NOCHL combination compared with V34 $(-3.2 \%$ and $-0.5 \%$ lower values for 242 Shannon and Simpson, respectively). This was mostly due to an increase in these indices for 243 V34 datasets after removal of plastid sequences, while they stayed stable for the NOCHL 244 datasets. Although not the main focus of this work, we noticed that bacterial communities retrieved from Ectocarpus were more diverse than those from the two Laminaria species.

246 This might be due to the nature of the samples as well as the extraction protocol where entire 247 algal specimens were ground for Ectocarpus (i.e. including the endomicrobiota), while for 248 Laminaria the extraction protocols only targeted surface-attached bacteria. Joint hierarchical 249 clustering of OTU-level datasets for both primer pairs after removal of plastid sequences 250 showed that samples grouped according to algal host rather than primer pair (Figure 3A).

251 The relative abundance of phyla was generally similar between paired sets of samples (Figure 252 3B). Notable exceptions were the Planctomycetes, Verrucomicrobia, and Cyanobacteria that 253 had lower relative abundance in the NOCHL libraries compared to V34 (Table S4). We 254 further searched for differential OTUs between pairs of samples sequenced with the V34 or 255 NOCHL combination. This analysis was performed with the edgeR package (Robinson et al., 256 2009) on the non-transformed dataset after removal of plastid sequences, and accounted for 257 the paired design of the study, i.e. the same samples were sequenced with the two primer 
258 combinations. The significance threshold was set at $\alpha=5 \%$ after Benjamini-Hochberg 259 correction for multiple testing. Out of the 2,852 OTUs remaining after discarding plastids, the 260 edgeR analysis detected 39 differential OTUs (i.e. 1.4\%) between primer combinations (Table

261 S5). All differential OTUs were more abundant in the V34-amplified dataset compared to 262 NOCHL. The majority of them belonged to Verrucomicrobiae and Planctomycetacia (Figure 263 4), partly reflecting the lower predicted coverage of NOCHL for these taxa. The apparent 264 lower abundance of Plantomycetacia OTUs using the NOCHL primer set could also be in 265 part due to their over-estimation with the V34 combination, as shown in the mock community 266 analysis (Table 2). The highest fold-change was found for OTU00082 belonging to 267 Cyanobacteria. The lower abundance of Cyanobacteria was inevitable since plastids and 268 extant cyanobacteria share a common ancestor and therefore have homologous 16S rDNA 269 sequences (Giovannoni et al., 1988; Delwiche, 1999).

\section{Conclusion}

272 Considering that plastid sequences can represent more than $90 \%$ of all sequences in one 273 sample when using universal primer sets, the bacterial diversity in a sample may be 274 underrepresented. The new primer set NOCHL is efficient to avoid amplifying plastid 275 sequences from an algal host while identifying a significantly higher bacterial richness than 276 with the universal primers V34, given identical sequencing efforts. This validates that fewer 277 plastid sequences in the samples lead to a larger access to bacterial sequences thus recovering 278 more of the bacterial diversity. However, as some bacterial groups may be underrepresented, 279 this primer set may be combined with taxon-specific primers (e.g. for Planctomycetes) for a 280 more complete coverage of Bacteria. Still, the data obtained with the NOCHL and universal 281 V34 primer sets are comparable since the targeted regions are the same and patterns of the community structure are similar. Although validated here on macroalgal samples, this primer 
283 set may be useful to characterize bacterial communities from other environments where

284 plastid contamination can be an issue such as terrestrial plants, microalgae, or gut microbiota 285 of herbivores.

\section{Acknowledgments and funding}

288 The authors have no conflict of interest to declare. We warmly thank Gaëlle Correc and 289 Sylvie Rousvoal for help with sampling as well as Florian de Bettignies and Dominique 290 Davoult for providing samples. We thank Frédérique Le Roux and Christian Jeanthon for 291 providing bacterial strains. This work has benefited from the facilities of the Genomer 292 platform and from the computational resources of the ABiMS bioinformatics platform (FR 293 2424, CNRS-Sorbonne Université, Roscoff), which are part of the Biogenouest core facility 294 network.

295 This work has benefited from the support of the French Government via the National 296 Research Agency investment expenditure program IDEALG (ANR-10-BTBR-04). FT, SD, 297 and CL acknowledge support by the Centre National de la Recherche Scientifique (CNRS). 298 AG acknowledges support by the Institut Français de Recherche pour l'Exploitation de la Mer 299 (IFREMER).

\section{References}

302 Aires, T., Muyzer, G., Serrão, E.A., and Engelen, A.H. (2018) Unraveling seaweeds 303 bacteriomes. In, Charrier,B., Wichard,T., and Reddy,C.R.K. (eds), Protocols for $304 \quad$ Macroalgae Research. CRC Press, pp. 95-113.

305 Aires, T., Serrão, E.A., and Engelen, A.H. (2016) Host and environmental specificity in bacterial communities associated to two highly invasive marine species (genus Asparagopsis). Front Microbiol 7: 1-14. 
308 Barott, K.L., Rodriguez-Brito, B., Janouškovec, J., Marhaver, K.L., Smith, J.E., Keeling, P., and Rohwer, F.L. (2011) Microbial diversity associated with four functional groups of benthic reef algae and the reef-building coral Montastraea annularis. Environ Microbiol 13: 1192-1204.

Bengtsson, M.M. and Øvreås, L. (2010) Planctomycetes dominate biofilms on surfaces of the kelp Laminaria hyperborea. BMC Microbiol 10: 261.

314 Bengtsson, M.M., Sjøtun, K., Lanzén, A., and Øvreås, L. (2012) Bacterial diversity in relation to secondary production and succession on surfaces of the kelp Laminaria hyperborea. ISME J 6: 2188-2198.

Bodenhausen, N., Horton, M.W., and Bergelson, J. (2013) Bacterial Communities Associated with the Leaves and the Roots of Arabidopsis thaliana. PLoS One 8:.

Burke, C., Thomas, T., Lewis, M., Steinberg, P., and Kjelleberg, S. (2011) Composition, uniqueness and variability of the epiphytic bacterial community of the green alga Ulva australis. ISME J 5: 590-600.

Chelius, M.K. and Triplett, E.W. (2001) The diversity of archaea and bacteria in association with the roots of Zea mays L. Microb Ecol 41: 252-263.

Delwiche (1999) Tracing the thread of plastid diversity through the tapestry of life. Am Nat 154: S164.

Dittami, S.M., Duboscq-Bidot, L., Perennou, M., Gobet, A., Corre, E., Boyen, C., and Tonon, T. (2016) Host-microbe interactions as a driver of acclimation to salinity gradients in brown algal cultures. ISME J 10: 51-63.

Egan, S., Fernandes, N.D., Kumar, V., Gardiner, M., and Thomas, T. (2014) Bacterial pathogens, virulence mechanism and host defence in marine macroalgae. Environ Microbiol 16: 925-938.

332 Egan, S., Harder, T., Burke, C., Steinberg, P., Kjelleberg, S., and Thomas, T. (2013) The 

seaweed holobiont: Understanding seaweed-bacteria interactions. FEMS Microbiol Rev 37: 462-476.

Gauthier, G., Gauthier, M., and Christen, R. (1995) Phylogenetic analysis of the genera Alteromonas, Shewanella, and Moritella using genes coding for small-subunit rRNA sequences and division of the genus Alteromonas into two genera, Alteromonas (emended) and Pseudoalteromonas gen. nov., and proposal of twelve new species combinations. Int J Syst Bacteriol 45: 755-761.

Giovannoni, S.J., Turner, S., Olsen, G.J., Barns, S., Lane, D.J., and Pace, N.R. (1988) Evolutionary relationships among cyanobacteria and green chloroplasts. J Bacteriol 170: $3584-3592$.

Hanshew, A.S., Mason, C.J., Raffa, K.F., and Currie, C.R. (2013) Minimization of chloroplast contamination in 16S rRNA gene pyrosequencing of insect herbivore bacterial communities. J Microbiol Methods 95: 149-155.

Illumina web page (2018) 16S Metagenomic Sequencing Library Preparation.

Klindworth, A., Pruesse, E., Schweer, T., Peplies, J., Quast, C., Horn, M., and Glöckner, F.O. (2013) Evaluation of general 16S ribosomal RNA gene PCR primers for classical and next-generation sequencing-based diversity studies. Nucleic Acids Res 41: 1-11.

Kozich, J.J., Westcott, S.L., Baxter, N.T., Highlander, S.K., and Schloss, P.D. (2013) Development of a dual-index sequencing strategy and curation pipeline for analyzing amplicon sequence data on the MiSeq Illumina sequencing platform. Appl Environ Microbiol 79: 5112-20.

Lachnit, T., Meske, D., Wahl, M., Harder, T., and Schmitz, R. (2011) Epibacterial community patterns on marine macroalgae are host-specific but temporally variable. Environ Microbiol 13: 655-665.

Lage, O.M. and Bondoso, J. (2014) Planctomycetes and macroalgae, a striking association. 
359 Lemay, M., Martone, P., Keeling, P., Burt, J., Krumhansl, K., Sanders, R., and Parfrey, L. (2018) Sympatric kelp species share a large portion of their surface bacterial communities. Environ Microbiol 20: 658-670.

van der Loos, L.M., Eriksson, B.K., and Falcão Salles, J. (2019) The macroalgal holobiont in a changing sea. Trends Microbiol 27: 635-650.

364 Miranda, L.N., Hutchison, K., Grossman, A.R., and Brawley, S.H. (2013) Diversity and abundance of the bacterial community of the red macroalga Porphyra umbilicalis: Did bacterial farmers produce macroalgae? PLoS One 8: e58269.

Paix, B., Othmani, A., Debroas, D., Culioli, G., and Briand, J.F. (2019) Temporal covariation of epibacterial community and surface metabolome in the Mediterranean seaweed holobiont Taonia atomaria. Environ Microbiol. doi: 10.1111/1462-2920.14617

Quast, C., Pruesse, E., Yilmaz, P., Gerken, J., Schweer, T., Yarza, P., et al. (2013) The SILVA ribosomal RNA gene database project: improved data processing and web-based tools. Nucleic Acids Res 41: D590-6.

Robinson, M.D., McCarthy, D.J., and Smyth, G.K. (2009) edgeR: A Bioconductor package for differential expression analysis of digital gene expression data. Bioinformatics 26: 139-140.

Sagaram, U.S., Deangelis, K.M., Trivedi, P., Andersen, G.L., Lu, S.E., and Wang, N. (2009) Bacterial diversity analysis of huanglongbing pathogen-infected citrus, using phyloChip arrays and 16S rRNA gene clone library sequencing. Appl Environ Microbiol 75: 15661574.

Serebryakova, A., Aires, T., Viard, F., Serrão, E.A., and Engelen, A.H. (2018) Summer shifts of bacterial communities associated with the invasive brown seaweed Sargassum muticum are location and tissue dependent. PLoS One 13: 1-18. 
383 Singh, R.P. and Reddy, C.R.K. (2016) Unraveling the functions of the macroalgal microbiome. Front Microbiol 6: 1-8.

385

Staufenberger, T., Thiel, V., Wiese, J., and Imhoff, J.F. (2008) Phylogenetic analysis of bacteria associated with Laminaria saccharina. FEMS Microbiol Ecol 64: 65-77.

Stratil, S.B., Neulinger, S.C., Knecht, H., Friedrichs, A.K., and Wahl, M. (2014) Salinity affects compositional traits of epibacterial communities on the brown macroalga Fucus vesiculosus. FEMS Microbiol Ecol 88: 272-279.

Stratil, S.B., Neulinger, S.C., Knecht, H., Friedrichs, A.K., and Wahl, M. (2013) Temperature-driven shifts in the epibiotic bacterial community composition of the brown macroalga Fucus vesiculosus. Microbiologyopen 2: 338-349.

Vieira, C., Engelen, A.H., Guentas, L., Aires, T., Houlbreque, F., Gaubert, J., et al. (2016) Species specificity of bacteria associated to the brown seaweeds Lobophora (Dictyotales, Phaeophyceae) and their potential for induction of rapid coral bleaching in Acropora muricata. Front Microbiol 7: 1-13.

Vollmers, J., Frentrup, M., Rast, P., Jogler, C., and Kaster, A.K. (2017) Untangling genomes of novel Planctomycetal and Verrucomicrobial species from Monterey bay kelp forest metagenomes by refined binning. Front Microbiol $\mathbf{8}$ :.

Wang, Q., Garrity, G.M., Tiedje, J.M., and Cole, J.R. (2007) Naive Bayesian classifier for rapid assignment of rRNA sequences into the new bacterial taxonomy. Appl Environ Microbiol 73: 5261-7.

Zozaya-Valdes, E., Egan, S., and Thomas, T. (2015) A comprehensive analysis of the microbial communities of healthy and diseased marine macroalgae and the detection of known and potential bacterial pathogens. Front Microbiol 6: 1-9. 


\section{$\underline{\text { Table and Figure legends }}$}

411 Figure 1: Relative abundance of sequences affiliated to plastid OTUs using the V34 or

412 NOCHL primer sets. Bacterial communities were analyzed in triplicate samples from 413 different algal hosts: ECTO, Ectocarpus subulatus; LHYP, Laminaria hyperborea; LDIG,

414 Laminaria digitata.

Figure 2: Richness and diversity estimates before (A) and after (B) removal of OTUs affiliated to plastids. Data were subsampled at 8,358 sequences per sample prior to analysis. Observed, observed OTU richness; Chao1, Chao 1 estimated richness; Shannon, Shannon index; Simpson, Simpson index; ECTO, Ectocarpus subulatus; LDIG, Laminaria digitata; LHYP, Laminaria hyperborea. Results of paired Student's t-test testing the effect of the set of primers on each measured estimate are reported below each panel $(\mathrm{df}=8)$.

Figure 3: Effect of the primer pair on the detected bacterial community composition, after removal of OTUs affiliated to plastids.

(A) Hierarchical clustering calculated on Hellinger-transformed data based on Morisita-Horn distance using the complete linkage algorithm

(B) Taxonomic composition based on the phylum level

Figure 4: Taxonomic affiliation of the 39 differential OTUs detected with edgeR, depending on the primer pair. All of these OTUs received higher coverage with the V34 primers.

431 Supplementary Figure 1: Rarefaction analysis of datasets obtained with V34 and NOCHL primer sets before (A) and after (B) removal of plastid-related sequences. 
434 Table 1: In silico predicted coverage of selected bacterial taxa, relative to the sequences

435 available in the Silva SSU r132 database for different primer combinations using TestPrime

436 1.0. A length of 3 bases of 0 -mismatch zone at 3' end of primers was chosen for tests with two

437 allowed mismatches in the sequence.

438

439 Table 2: Genus-level comparison of theoretical relative abundance in the mock community

440 with the measured abundance in libraries sequenced with V34 or NOCHL primers. unclass.,

441 unclassified.

442 


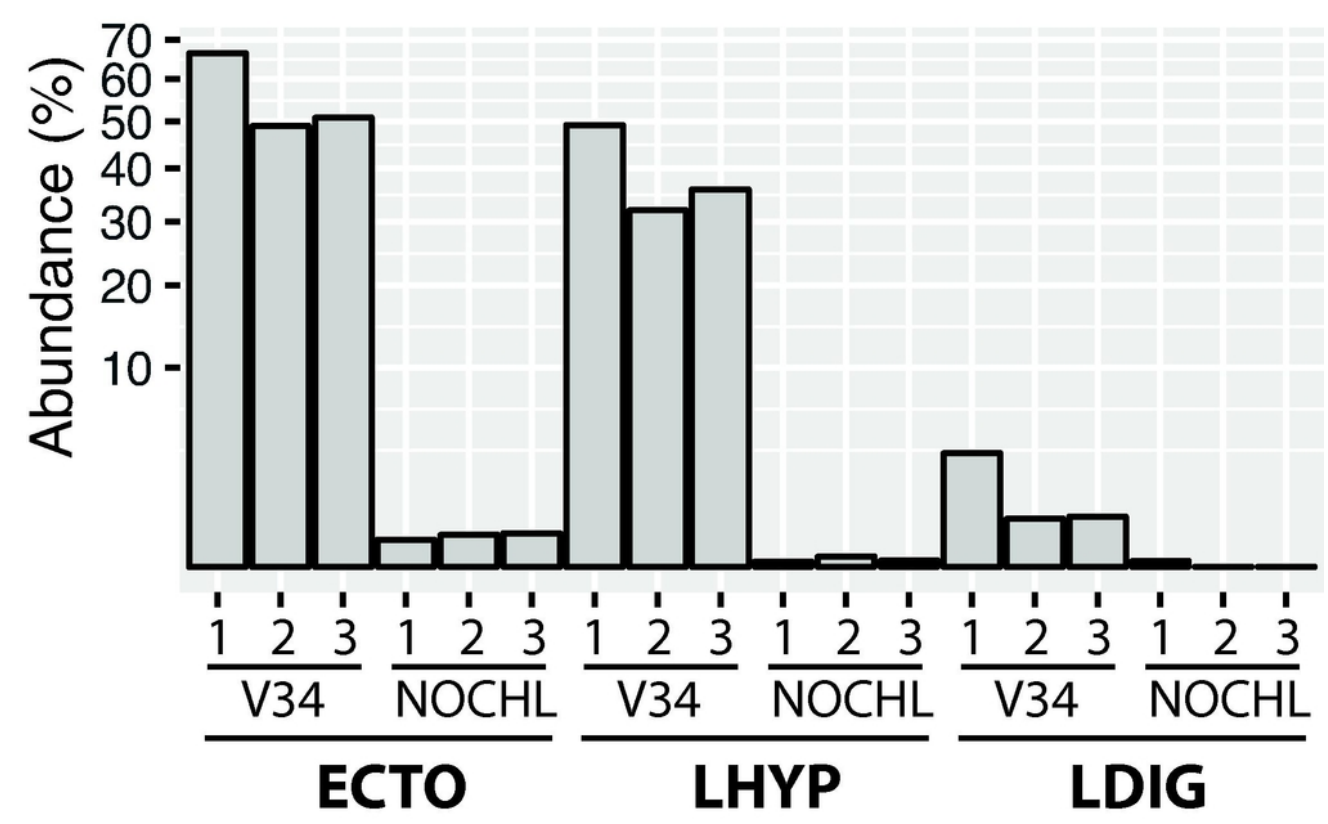

Figure 1: Relative abundance of sequences affiliated to plastid OTUs using the V34 or NOCHL primer sets. Bacterial communities were analyzed in triplicate samples from different algal hosts: ECTO, Ectocarpus subulatus; LHYP, Laminaria hyperborea; LDIG, Laminaria digitata.

$$
85 \times 53 \mathrm{~mm}(300 \times 300 \mathrm{DPI})
$$


A.

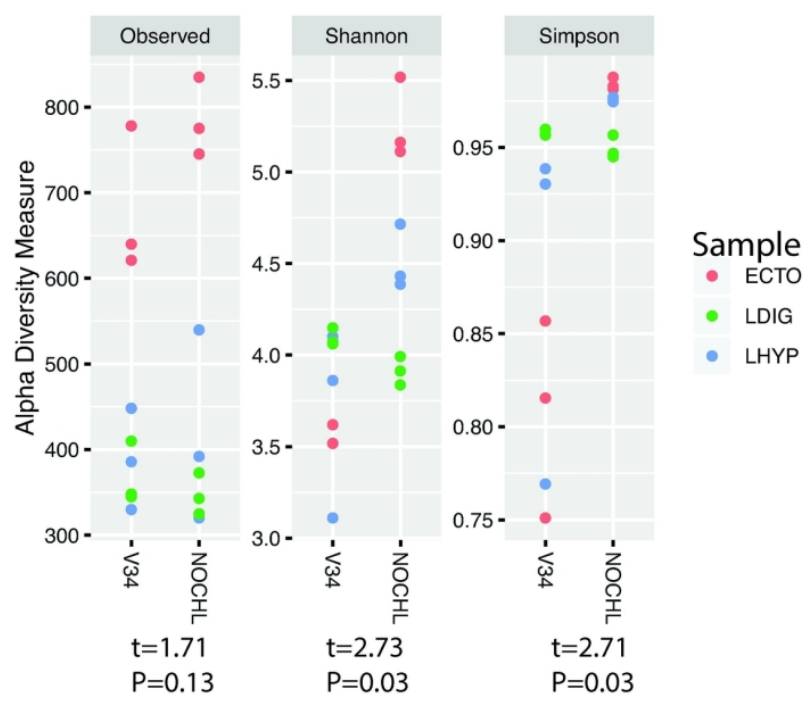

B.

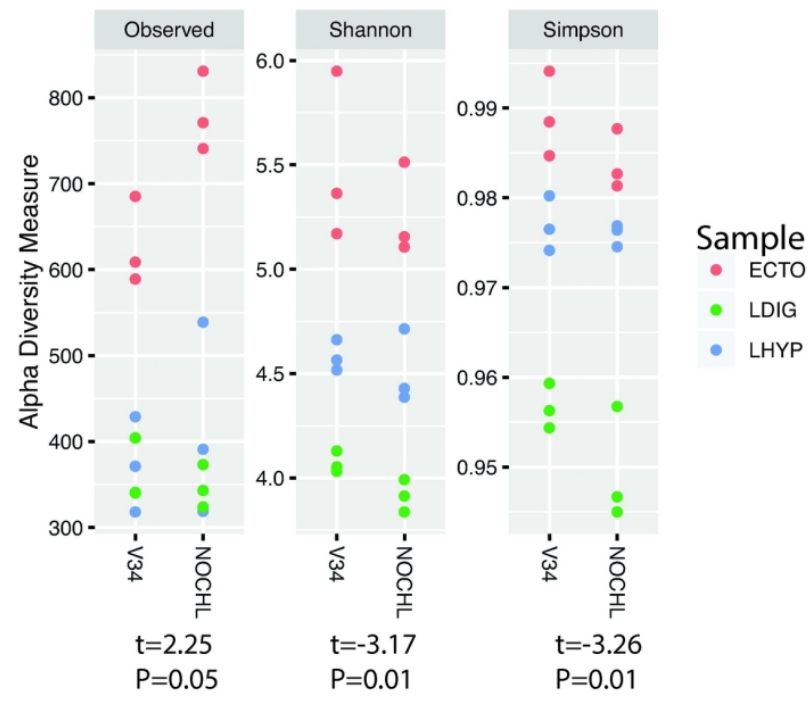

Figure 2: Richness and diversity estimates before (A) and after (B) removal of OTUs affiliated to plastids. Data were subsampled at 8,358 sequences per sample prior to analysis. Observed, observed OTU richness; Shannon, Shannon index; Simpson, Simpson index; ECTO, Ectocarpus subulatus; LDIG, Laminaria digitata; LHYP, Laminaria hyperborea. Results of paired Student's t-test testing the effect of the set of primers on each measured estimate are reported below each panel $(\mathrm{df}=8)$.

$111 \times 188 \mathrm{~mm}(300 \times 300 \mathrm{DPI})$ 
A.

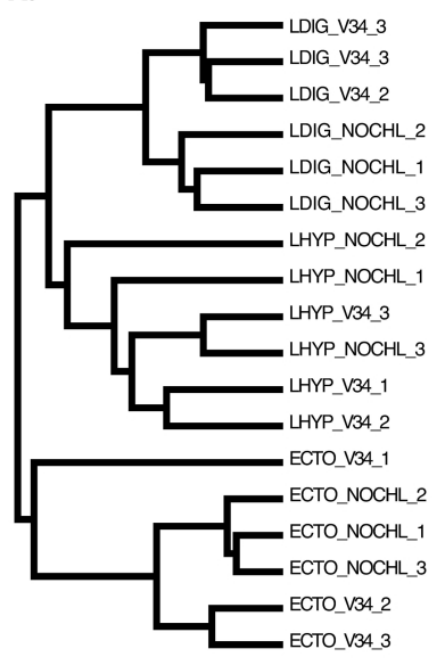

$\overline{0.05}$
B.

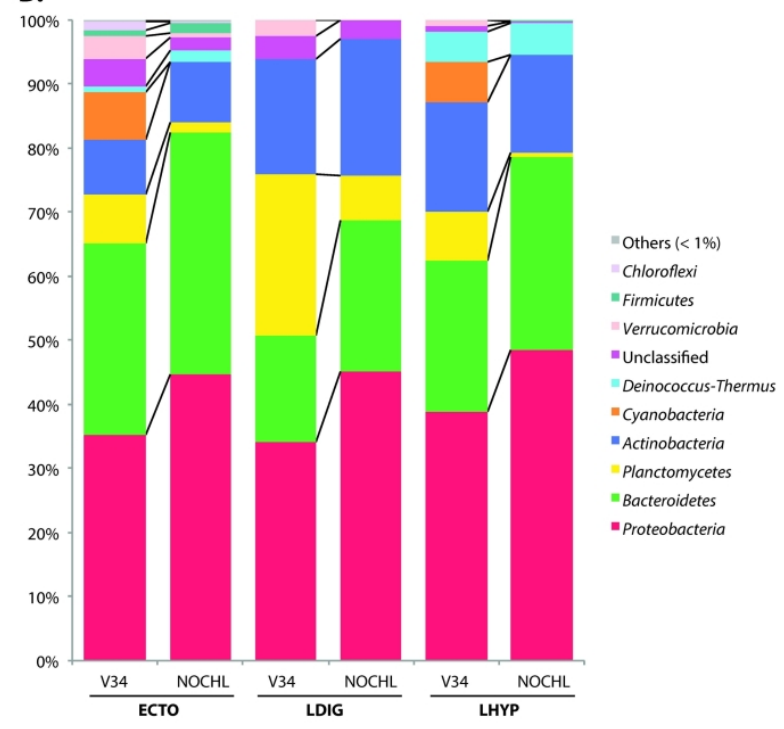

Figure 3: Effect of the primer pair on the detected bacterial community composition, after removal of OTUs affiliated to plastids.

(A) Hierarchical clustering calculated on Hellinger-transformed data based on Morisita-Horn distance using the complete linkage algorithm

(B) Taxonomic composition based on the phylum level

$271 \times 164 \mathrm{~mm}(300 \times 300 \mathrm{DPI})$ 


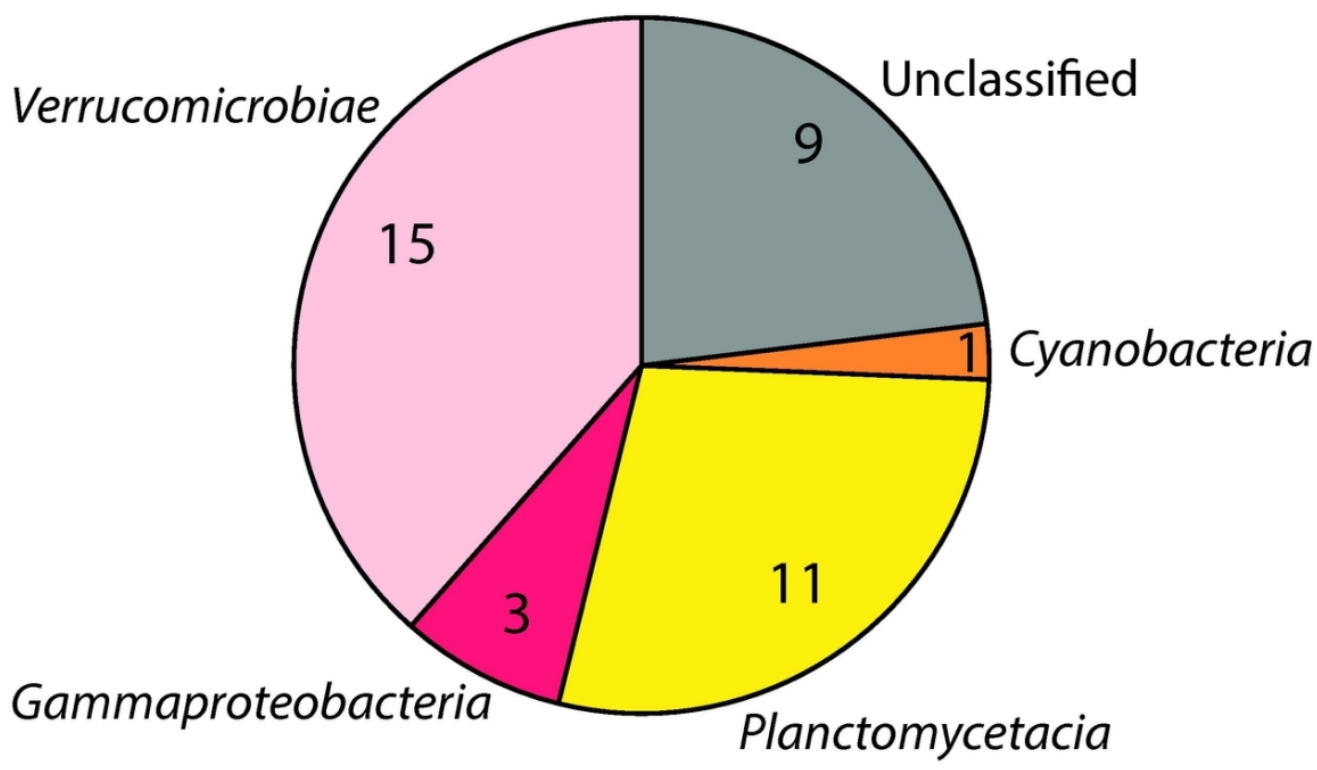

Figure 4: Taxonomic affiliation of the 39 differential OTUs detected with edgeR, depending on the primer pair

$105 \times 76 \mathrm{~mm}(300 \times 300 \mathrm{DPI})$ 
Table 1: In silico predicted coverage of selected bacterial taxa, relative to the sequences available in the Silva SSU r132 database for different primer combinations using TestPrime 1.0. A length of 3 bases of 0 -mismatch zone at 3 ' end of primers was chosen for tests with two allowed mismatches in the sequence.

\begin{tabular}{|c|c|c|c|c|c|c|c|c|c|c|}
\hline & \multicolumn{5}{|c|}{0 mismatch } & \multicolumn{5}{|c|}{2 mismatches } \\
\hline & V34 & NOCHL & NOCHL3 & NOCHL6 & NOCHL7 & V34 & NOCHL & NOCHL3 & NOCHL6 & NOCHL7 \\
\hline Acidobacteria & 92.0 & 43.1 & 43.2 & 43.1 & 43.3 & 95.7 & 95.0 & 45.3 & 45.1 & 96.2 \\
\hline Actinobacteria & 82.4 & 82.5 & 82.7 & 82.6 & 82.7 & 86.6 & 96.3 & 96.5 & 96.1 & 86.9 \\
\hline Aquificae & 89.3 & 89.6 & 90.2 & 89.9 & 90.5 & 95.0 & 95.5 & 95.8 & 95.5 & 96.1 \\
\hline Bacteroidetes & 89.8 & 88.8 & 89.3 & 89.0 & 89.4 & 95.4 & 95.8 & 95.2 & 94.8 & 95.9 \\
\hline Chlamydiae & 82.8 & 65.9 & 65.9 & 65.9 & 66.4 & 96.7 & 96.9 & 79.7 & 79.7 & 96.7 \\
\hline Chloroflexi & 39.0 & 19.3 & 20.8 & 20.7 & 21.1 & 88.7 & 48.6 & 30.3 & 30.3 & 88.6 \\
\hline Cyanobacteria & 76.2 & 0.6 & 0.6 & 0.6 & 0.6 & 92.4 & 1.9 & 0.9 & 0.9 & 88.6 \\
\hline Chloroplasts & 57.3 & 0.7 & 0.7 & 0.7 & 0.7 & 88.3 & 2.7 & 1.1 & 1.1 & 81.3 \\
\hline $\begin{array}{l}\text { Deinococcus- } \\
\text { Thermus }\end{array}$ & 92.9 & 92.6 & 92.6 & 92.6 & 93.0 & 96.7 & 96.9 & 96.3 & 96.2 & 96.7 \\
\hline Epsilonbacteraeota & 93.9 & 85.1 & 85.9 & 85.5 & 85.8 & 96.8 & 96.6 & 88.6 & 88.2 & 97.3 \\
\hline Firmicutes & 88.2 & 84.0 & 84.9 & 84.7 & 85.0 & 94.2 & 92.8 & 90.5 & 90.1 & 94.7 \\
\hline Fusobacteria & 87.1 & 86.1 & 87.0 & 86.7 & 87.2 & 94.9 & 94.0 & 93.9 & 93.6 & 95.3 \\
\hline Gemmatimonadetes & 89.6 & 89.2 & 89.8 & 89.4 & 89.8 & 93.6 & 94.7 & 95.5 & 94.4 & 94.9 \\
\hline Planctomycetes & 73.5 & 10.6 & 10.6 & 10.5 & 10.7 & 85.1 & 82.3 & 12.0 & 11.9 & 85.8 \\
\hline Proteobacteria & 90.0 & 89.0 & 89.4 & 89.2 & 89.4 & 95.7 & 94.3 & 93.6 & 93.1 & 96.2 \\
\hline Spirochaetes & 75.7 & 70.5 & 70.7 & 70.3 & 70.7 & 88.2 & 94.6 & 85.6 & 85.1 & 88.4 \\
\hline Verrucomicrobia & 87.3 & 16.2 & 16.3 & 16.3 & 16.5 & 93.0 & 48.7 & 17.2 & 17.1 & 92.5 \\
\hline All Bacteria & 86.6 & 79.1 & 79.6 & 79.3 & 79.6 & 93.6 & 90.1 & 85.3 & 84.9 & 93.9 \\
\hline
\end{tabular}


Table 2: Comparison between the theoretical and the observed relative abundance of genera in the mock community in libraries sequenced with V34 or NOCHL primers. unclass., unclassified.

\section{Relative abundance of sequences}

\section{Genus}

in the mock community (\%)

\begin{tabular}{|c|c|c|c|}
\hline & Theory & $\begin{array}{l}\text { Primers } \\
\text { V34 }\end{array}$ & $\begin{array}{l}\text { Primers } \\
\text { NOCHL }\end{array}$ \\
\hline Vibrio & 24.63 & 8.28 & 8.78 \\
\hline Formosa & 9.72 & 3.04 & 2.53 \\
\hline Maribacter & 9.42 & 5.41 & 5.24 \\
\hline Zobellia & 7.24 & 5.07 & 3.04 \\
\hline Alteromonas & 5.29 & 1.01 & 1.35 \\
\hline Nonlabens & 5.02 & 0.00 & 0.00 \\
\hline Psychrobacter & 2.59 & 6.08 & 4.05 \\
\hline Microbacterium & 2.79 & 0.51 & 0.34 \\
\hline Agrococcus & 2.74 & 0.00 & 0.00 \\
\hline Sphingomonas & 2.51 & 1.52 & 2.20 \\
\hline Arthrobacter & 2.42 & 0.00 & 0.00 \\
\hline Dokdonia & 2.35 & 0.00 & 0.00 \\
\hline Paracoccus & 2.31 & 2.87 & 4.22 \\
\hline Polaribacter & 2.15 & 8.78 & 9.12 \\
\hline Roseovarius & 1.94 & 0.00 & 0.00 \\
\hline Cobetia & 1.94 & 5.24 & 6.25 \\
\hline Winogradskyella & 1.91 & 1.35 & 2.03 \\
\hline Roseobacter & 1.90 & 0.00 & 1.35 \\
\hline Cellulophaga & 1.77 & 3.38 & 2.53 \\
\hline Mariniflexile & 1.74 & 1.86 & 1.01 \\
\hline Pseudoalteromonas & 1.71 & 11.82 & 11.99 \\
\hline Hoeflea & 1.57 & 0.00 & 1.86 \\
\hline Bosea & 1.30 & 0.68 & 0.84 \\
\hline Imperialibacter & 1.23 & 0.00 & 0.00 \\
\hline Rhodopirellula & 1.15 & 1.69 & 0.51 \\
\hline Dinoroseobacter & 0.68 & 0.51 & 0.17 \\
\hline unclass. Acidimicrobiales & - & 0.34 & 0.00 \\
\hline unclass. Vibrionaceae & - & 11.49 & 10.81 \\
\hline unclass. Sphingobacteriales & - & 5.24 & 6.93 \\
\hline unclass. Micrococcaceae & - & 3.89 & 3.04 \\
\hline unclass. Flavobacteriaceae & - & 5.41 & 4.05 \\
\hline unclass. Rhodobacteraceae & - & 1.52 & 3.21 \\
\hline Krokinobacter & - & 1.86 & 2.03 \\
\hline unclass. Gammaproteobacteria & - & 0.34 & 0.34 \\
\hline unclass. Actinobacteria & - & 0.34 & 0.00 \\
\hline unclass. Proteobacteria & - & 0.17 & 0.00 \\
\hline unclass. Alteromonadaceae & - & 0.00 & 0.17 \\
\hline unclass Hyphomonadaceae & - & 0.34 & 0.00 \\
\hline $\begin{array}{l}\text { Correlation coefficient Theory vs. Observed } \\
\text { for genera present in the mock community }\end{array}$ & & 0.39 & 0.38 \\
\hline
\end{tabular}

